\title{
Travelling Wave Solution of a Riemann Problem and Shock Structure in an Unsteady Flow of a Perfect Gas under Viscosity
}

\author{
Manoj Singh, Arvind Patel*, Ruchi Bajargaan \\ Department of Mathematics, University of Delhi, Delhi 110007, India
}

Corresponding Author Email: arvindpatelmath09@gmail.com

https://doi.org/10.18280/ijht.370329

Received: 14 January 2018

Accepted: 17 July 2019

\section{Keywords:}

Navier-Stokes equations, Perfect gas, Riemann condition, Shock wave, Viscosity

\begin{abstract}
Travelling wave solution of one-dimensional unsteady flow of a perfect gas with the effect of viscosity under Riemann condition is investigated. The system of gas dynamic equations are reduced into a single ordinary differential equation for non-dimensional velocity and Riemann condition is transformed into boundary conditions. The exact solution is obtained for the gas velocity, pressure, temperature and change-in-entropy under the constant boundary conditions taking the origin at inflection point of the gas velocity profile. It is found that the travelling wave is a shock transition zone of the thickness of order $10^{\wedge}(-6)$ meter. The viscosity of the gas, Mach number, ratio of specific heats, and Riemann condition has significant effects on the shock structure.
\end{abstract}

\section{INTRODUCTION}

Travelling wave solution was first considered in 1930s by Fisher [1] and Kolmogorov, Petrovsky, and Piscounoff [2] for what has been now known as the Fisher-KPP equation. After that, travelling wave solutions have been extensively used in the solution of the many physical problems. Travelling wave solutions of reaction-diffusion equations have been extensively covered in [3]. Travelling wave solutions of a nonlinear reaction-diffusion equations in chemotaxis model for bacterial pattern formation was studied by Mamsour [4]. Apreutesei [5] has studied the existence of travelling waves solutions of two Atherosclerosis models presented in terms of reaction-diffusion equations in an infinite strip with nonlinear boundary conditions. Marchant, Norbury and Perumpanani [6] have studied travelling shock waves arising in a model of malignant invasion. Marchant, Norbury, and Sherrantt [7] have obtained the travelling wave solutions to a haptotaxis-dominated model of malignant invasion. Travelling wave phenomena for viscoelastic generalization of Burger's equation was presented by Camacho, Guy and Jacobsen [8]. In 2015, Eabay and Sengul [9] have studied travelling waves in one-dimensional nonlinear models of strain-limiting viscoelasticity. In 2019, Roshid and Bashar [10] have used simple equation method based on traveling wave to find the breather wave and kinky periodic wave solutions of one-dimensional Oskolkov equation.

The Riemann problem consists of hyperbolic partial differential equation together with the discontinuous initial data. The Riemann problem for a system of conversation laws in gas dynamics, shallow water theory and magnetohydrodynamic has attracted considerable attention of the researchers for its theoretical and numerical solution. The first existence theorem for the solutions to the initial value problem for non-linear hyperbolic systems of equations was given by Glimm [11] in this fundamental paper. Courant and
Friedrichs [12] have given the Riemann problem corresponding to the shock-tube problem for Euler's equations, which is a basic physical problem in gas dynamics. Lax [13] solved the Riemann problem with sufficiently close left and right initial data separated at the origin.

The Riemann problem for Euler's equations does not admit a closed form solution even for an ideal gas. Several researchers have been motivated to develop iterative schemes to determine the solution of such problems. Godunov [14] had proposed two methods based on a fixed point scheme. Smoller [15] had given a different approach in which solution was derived in the implicit form for an ideal gas. Roe [16] developed a Riemann solver for an ideal polytropic gas. The Riemann problem was also attempted by Colella [17], for real gases using Riemann solvers. Recently in 2016, Ambika and Radha [18] have studied the Riemann problem for gas dynamic equations governing a one-dimensional flow of a van der Waals gases. The solution of Riemann problem has given rise the shock, contact discontinuity or rarefaction waves depending on the different initial data.

The study of the structure of shock waves have been a challenging field of investigation for theoreticians and experimental scientists for a long time. In order to understand the internal structure of a shock wave, it is required to investigate the rapid change of flow variables such as velocity, pressure, temperature, change-in-entropy, viscous stress, heat flux, shock asymmetry, and ratio of mean free path to the thickness of the shock wave, through a narrow region between the two equilibrium states. There is a rich history of theoretical and experimental study of the shock wave structure.

The structure of a shock wave has been studied extensively for propagation of a perfect gas in a viscous steady flow in presence or absence of heat conduction. Rankine [19], Rayleigh [20], and Taylor [21] have started the investigation of dissipative process due to the viscosity and heat 
conduction. Later, Lamb [22], Becker [23], Thomas [24], Meyerhoff and Reissner [25], Puckett [26], Thompson and Lambrakis [27], Cramer and Crickenberger [28], Johnson [29], Anand and Yadav [30, 31] have studied the shock structure problem in ideal or non-ideal gas under the presence or absence of viscosity and heat conductivity by different approach. In 2014, Myong [32] has investigated analytical solutions of the shock structure equations and asymmetry in the frame work of Navier-Stokes and Fourier equations in a perfect gas for Prandtl number $\mathrm{Pr}=3 / 4$. Recently, Patel and Singh [33] have studied shock wave structure in a non-ideal gas under constant and vaiable coefficients of viscosity and heat conductivity in a steady flow. Most of the above studies were confined under the assumption of steady flow condition.

In 2016, Singh, Patel and Bajargaan [34] have studied the unsteady one-dimensional gas dynamic problem by Adomian decomposition method. They have been successful to capture the shock wave in their solution for planer, cylindrical and spherical symmetries but were unable to investigate the internal structure of shock waves. In 2017, Bajargaan, Patel, and Singh [35] solved the system of gas dynamic equation for unsteady flow of an ideal gas by homotopy analysis method and obtained the shock phenomena but were not able to obtain structure of shock waves. The most general and practical approach will be that in which the complete internal shock structure can be investigated for an unsteady flow of a gas.

In the present work, the method of travelling wave solution is employed to study the unsteady viscous and perfect gas flow under the Riemann condition. The Riemann problem is considered for arbitrary initial data and a condition is derived on Riemann data for existence of solution in terms of shocks. It is assumed, that the heat conduction and radiation heat flux in the fluid flow are negligible. The system of unsteady gas dynamic equations are reduced into a single first order ordinary differential equation in term of non-dimensional velocity and the Riemann condition is transformed into the boundary conditions at $\pm \infty$ for finite time, Thus obtained boundary value problem is solved for exact solution of the velocity, the pressure, the temperature and the change in entropy by setting the origin of co-ordinate system at the inflection point of the velocity profile. The RankineHugoniot jump conditions are derived in due process of exact solution and Lax entropy condition is used for the study of shock wave. The structure of a shock wave for unsteady flow of a perfect gas is investigated in terms of shock thickness, Mach number, viscosity, ratio of specific heat of the gas, and inverse shock thickness. The strength of shock wave is also discussed. All the numerical estimation of flow variables is carried out using Mathematica 9.

\section{BASIC EQUATIONS AND BOUNDARY CONDITION}

The gas dynamic equations governing the one-dimensional unsteady flow of perfect gas under the effect of viscosity can be expressed conveniently in Eularian coordinates in absence of body force for planar geometry as follows [18, 30, 31]

$$
\begin{gathered}
\frac{\partial \rho}{\partial t}+\frac{\partial(\rho u)}{\partial x}=0, \\
\frac{\partial(\rho u)}{\partial t}+\frac{\partial\left(p+\rho u^{2}-q\right)}{\partial x}=0,
\end{gathered}
$$

$$
\frac{\partial\left(\rho e+\frac{\rho u^{2}}{2}\right)}{\partial t}+\frac{\partial\left(\rho u\left(e+\frac{u^{2}}{2}\right)+p u-q u\right)}{\partial x}=0,
$$

where, $\rho(x, t), u(x, t), p(x, t), q(x, t)$ and $e(x, t)$ are the density, gas velocity, pressure, viscous stress and internal energy per unit mass respectively and, $x$ and $t$ are space the time co-ordinate. The viscous stress $(\mathrm{q})$ is given by

$$
q=\frac{4}{3} \mu \frac{\partial u}{\partial x}
$$

where, $\mu$ is the coefficient of dynamic viscosity (in limit of negligible bulk viscosity), which is assumed to be independent of the temperature and density for the simplicity. The solution of equations (1)-(3) has to be investigated by the method of travelling wave solution under the Riemann condition

$$
(\rho, u, p)(x, 0)= \begin{cases}\left(\rho_{1}, u_{1}, p_{1}\right), & x<0 \\ \left(\rho_{2}, u_{2}, p_{2}\right), & x>0\end{cases}
$$

which show a discontinuity in initial data at $x=0$. The initial data $\left(\rho_{1}, u_{1}, p_{1}\right)$ and $\left(\rho_{2}, u_{2}, p_{2}\right)$ are arbitrary constants that includes the values at $x \rightarrow \pm \infty$.

Equation of state of perfect gas is taken in the form

$$
p=\rho R T,
$$

where, $\mathrm{R}$ is the gas constant and $\mathrm{T}$ is the absolute temperature of the gas. The internal energy per unit mass of the perfect gas is given by

$$
e=C_{v} T=\frac{p}{\rho(\gamma-1)}
$$

where, $C_{v}=R /(\gamma-1)$ is the specific heat at constant volume and $\gamma$ is the ratio of specific heats $C_{p}$ at constant pressure and $C_{v}$ at constant volume.

\section{TRAVELING WAVE ANALYSIS}

In this section, we have given the travelling wave analysis of the system of one-dimensional unsteady gas dynamic equations (1)-(4) under Riemann condition (5). For, we have assumed that the solution of the Eqns. (1)-(4) is given by

$$
\begin{gathered}
u(x, t)=u(\xi), \rho(x, t)=\rho(\xi), p(x, t)=p(\xi), \\
\xi=x-c t
\end{gathered}
$$

where, $\mathrm{c}$ is a constant speed of right travelling wave to be determined later and is a wave variable. Under the transformation (8), the Eqns. (1)-(4) reduces into

$$
\begin{gathered}
c \frac{d \rho}{d \xi}-\frac{d(\rho u)}{d \xi}=0, \\
c \frac{d(\rho u)}{d \xi}-\frac{d\left(p+\rho u^{2}-q\right)}{d \xi}=0, \\
c \frac{d\left(\rho e+\rho u^{2} / 2\right)}{d \xi}-\frac{d\left(\rho u\left(e+u^{2} / 2\right)+p u-q u\right)}{d \xi}=0, \\
q=\frac{4}{3} \mu \frac{d u}{d \xi} .
\end{gathered}
$$


Thus, the method of travelling wave solution reduces the system of gas dynamic Eqns. (1)-(4) from partial differential equations to exact ordinary differential equations. Now Eqns. (9)-(11) can be integrated with respect to $\xi$ to give

$$
\begin{gathered}
\rho(c-u)=\rho_{1}\left(c-u_{1}\right)=A \\
p-\rho\left(c^{2}-u^{2}\right)-q=p_{1}-\rho_{1}\left(c^{2}-u_{1}{ }^{2}\right)=B \\
(c-u)\left\{\frac{p}{\gamma-1}+\frac{\rho u^{2}}{2}\right\}-p u+q u=\left(c-u_{1}\right)\left\{\frac{p_{1}}{\gamma-1}+\frac{\rho_{1} u_{1}{ }^{2}}{2}\right\}- \\
p_{1} u_{1}=C
\end{gathered}
$$

where, $\mathrm{A}, \mathrm{B}$ and $\mathrm{C}$ are constants. The viscous stress tenser $\mathrm{q}$ in the left hand side of the equations (14)-(15) is given by Eq. (12) and is equal to zero at $\xi \rightarrow \pm \infty$. Therefore, from the Eqns. (13)-(15), we get a jump relation among the flow variables under the Riemann conditions (5) as

$$
\begin{gathered}
\rho_{2}\left(c-u_{2}\right)=\rho_{1}\left(c-u_{1}\right), \\
p_{2}-\rho_{2}\left(c^{2}-u_{2}{ }^{2}\right)=p_{1}-\rho_{1}\left(c^{2}-u_{1}{ }^{2}\right), \\
\left(c-u_{2}\right)\left\{\frac{p_{2}}{\gamma-1}+\frac{\rho_{2} u_{2}{ }^{2}}{2}\right\}-p_{2} u_{2}=\left(c-u_{1}\right)\left\{\frac{p_{1}}{\gamma-1}+\frac{\rho_{1} u_{1}{ }^{2}}{2}\right\}- \\
p_{1} u_{1},
\end{gathered}
$$

which are the Rankine-Hugoniot jump conditions across the discontinuity propagating with speed $\mathrm{c}$. These relations show that the discontinuity in the initial data at $x=0$, propagates with speed $\mathrm{c}$ into the flow-field. This jump discontinuity can be identified as a shock moving with constant speed c. Therefore, the right travelling wave in the Eq. (8) with the constant speed $\mathrm{c}$ is actually a shock wave. The profile for the gas velocity, temperature, pressure and change in entropy can be obtained from the Eqns. (12)-(15). This problem may be seen as a shock structure problem. Using Eqns. (12), (13) and (14) into the Eq. (15), the shock structure problem reduces into a single first order ordinary differential equation as

$$
\left(\frac{c-u}{\gamma-1}\right) \frac{4}{3} \mu \frac{d u}{d \xi}+\left(\frac{c-\gamma u}{\gamma-1}\right)(A u+A c+B)+\frac{A u^{2}}{2}=C,
$$

where, the constant A, B and C are given by Eqns. (13)-(15) and are the known function of the constant state $\left(\rho_{1}, u_{1}, p_{1}\right)$ at $\xi \rightarrow-\infty$. The trans-formation of initial condition (5) in term of wave variable $\xi$ has been given in section 4 .

\section{EXACT SOLUTIONS}

For the exact solution of Eq. (19), we introduce the nondimensional velocity $\eta$ and Mach number $M_{1}$ as

$$
\eta=\frac{c-u}{c-u_{1}}=\frac{\rho_{1}}{\rho}, \quad M_{1}=\frac{u_{1}-c}{a_{1}}
$$

where, the shock speed $\mathrm{c}$ is so chosen that the Lax-entropy condition $u_{1}>c>u_{2}$ for initial condition $u_{1}>u_{2}$, is satisfied and $a_{1}{ }^{2}=\gamma p_{1} / \rho_{1}$ is the speed of the sound at the uniform state at $\xi=-\infty$. Further, we can discuss the solution of Eq. (19) under the entropy condition $u_{1}<c<u_{2}$ for $u_{1}<$ $u_{2}$ as a separate case. In this case the initial Mach number will be taken as $M_{1}=\left(c-u_{1}\right) / a_{1}$. For the existence of shock, the flow speed $u$ must be decreases with an increase in the position $\xi$, therefore, we have chosen the initial data $u_{1}=3 c / 2$ and $u_{2}=c / 2$ so that the Lax entropy condition $u_{1}>c>u_{2}$ must hold (see [37]). We may be chosen other possible values for $u_{1}$, and $u_{2}$ in term of shock speed c under the Lax-entropy condition. We may determine the value of $c$ from the second Eq. of (20) for initial value $u_{1}$ and Mach number $M_{1}$. The governing Eq. (19) under the transformation (20) reduces into

$$
\frac{d \eta}{d \xi}=\frac{D \eta^{2}+E \eta+F}{G \eta}
$$

where,

$$
\begin{gathered}
D=-\frac{A(\gamma+1)\left(c-u_{1}\right)^{2}}{2}, \\
E=\gamma\left(c-u_{1}\right)(B+2 A c), \\
F=(1-\gamma)\left(\frac{3 A c^{2}}{2}+B c+C\right), G=\frac{4}{3} \mu\left(c-u_{1}\right)^{2} .
\end{gathered}
$$

Now, we transform the initial data for velocity given in Eq. (5) in term of non-dimensional velocity $\eta$ as a function of wave variables $\xi=x-c t$. It is observed that, for finite time $\mathrm{t}$ (including initial time $\mathrm{t}=0$ ), $\xi=x-c t \rightarrow \pm \infty$ as $x \rightarrow \pm \infty$. Therefore, using the wave variable $\xi$, the initial conditions at the boundary will be transform into boundary conditions. This is justifiable on the basis that discontinuity in initial data at $x=0$ shall affect the value of initial condition at $\pm \infty$ after some finite interval of time. Using this argument, the initial condition

$$
u(x, 0)= \begin{cases}u_{1}, & x<0 \\ u_{2}, & x>0\end{cases}
$$

under the Eq. (20) is transformed into boundary condition

$$
\eta(\xi)= \begin{cases}1, & \xi \rightarrow-\infty \\ -1, & \xi \rightarrow \infty\end{cases}
$$

Now, we shall obtain the solution of Eq. (21) under the boundary condition (22). Since, $\eta$ is constant as $\xi$ tends to $\pm \infty$, therefore, for large values of $\xi, \mathrm{d} \eta / \mathrm{d} \xi$ must be equal to zero. Using $d \eta / d \xi=0$ into the Eq. (21), we get a quadratic equation in $\eta$. The two roots $\eta_{1}$ and $\eta_{2}$ of the quadratic equation gives the actual boundary states between which the solution of the Eq. (21) exist. The two boundary states $\eta_{1}$ and $\eta_{2}$ are given by

$$
\eta_{1,2}=\frac{-E \pm \sqrt{\left(E^{2}-4 D F\right)}}{2 D} .
$$

It is found that the value of one of the roots of $\eta_{1}$ and $\eta_{2}$ is 1 which is exactly same as the value of $\eta$ at $\xi \rightarrow-\infty$ into the Eq. (22). This root remains same for all possible values of initial condition $u_{1}$ from Eqns. (20) and (23). The value of $\eta$ at $\xi \rightarrow+\infty$ into Eq. (22) and the other root in Eq. (23) depends on the choice of initial condition $u_{1}$ and $u_{2}$.

On integration of Eq. (21) by the method of partial fraction, using $\eta_{1}$ and $\eta_{2}$ from Eq. (23), we get $\xi$ in closed form as

$$
\xi=A_{1} \log \left(\eta-\eta_{1}\right)+B_{1} \log \left(\eta-\eta_{2}\right)+C^{\prime},
$$

where, 
$A_{1}=\frac{G \eta_{1}}{D\left(\eta_{1}-\eta_{2}\right)}, B_{1}=\frac{G \eta_{2}}{D\left(\eta_{2}-\eta_{1}\right)}$, and $C^{\prime}$ is the constant of integration. The value of constant $C^{\prime}$, can be obtained by choosing the origin $(\xi=0)$ of the coordinate system at the inflection point of the velocity profile $\eta$, which is given by $d^{2} \eta / d \xi^{2}=0$. The equation (21) yields a quadratic equation under the condition $d^{2} \eta / d \xi^{2}=0$ at $\xi=0$ given as

$$
D \eta_{\text {in }}{ }^{2}-F=0 \text {, }
$$

where, $\eta_{\text {in }}$ is value of velocity $\eta$ at inflection point. The solution of equation (25) gives

$$
\eta_{\text {in }}= \pm \sqrt{\frac{F}{D}}
$$

Among the two possible roots, let us assume that $\eta_{i n}=\eta^{*}$ is the maximum value of the real root $\eta_{i n}$ at the inflection point $\xi=0$.

The $\xi=x-c t=0$ corresponds to the two following cases:

$x=0, t=0$ gives the position of discontinuity into the initial data given by Riemann condition.

(ii) $x=c t$ gives the position of moving discontinuity with speed $c$ at time $t$.

Using $\eta=\eta^{*}$ at $\xi=0$ into the equation (24), we have

$$
C^{\prime}=-\left[A_{1} \log \left(\eta^{*}-\eta_{1}\right)+B_{1} \log \left(\eta^{*}-\eta_{2}\right)\right] .
$$

Therefore, the exact solution for the gas velocity with respect to the position is obtained in implicit form as

$$
\xi=A_{1} \log \left[\frac{\eta-\eta_{1}}{\eta^{*}-\eta_{1}}\right]+B_{1} \log \left[\frac{\eta-\eta_{2}}{\eta^{*}-\eta_{2}}\right] .
$$

This relation gives the transition of velocity from one uniform state $\eta=\eta_{1}=1$ at $\xi=-\infty$ to other $\eta=\eta_{2}$ at $\xi=$ $+\infty$ through the inflection point $\eta_{\text {in }}=\eta^{*}$. This proves the existence of transition zone in unsteady flow of perfect gas together with the effect of viscosity under Riemann condition.

Using Eqns. (12), (13), (20), and (21) into Eq. (14), we can obtain the exact solution for the distribution of pressure across the shock transition region as

$$
\frac{p}{p_{1}}=1+\frac{A^{2}(1-\eta)}{p_{1} \rho_{1}}-\frac{\rho_{1}\left(D \eta^{2}+E \eta+F\right)}{A p_{1} \eta}
$$

The Eq. (28) gives a relation between the pressure and the gas velocity. Thus, Eqns. (27) and (28) together give the variation of the pressure across the shock front with respect to the position $\xi$.

Similarly, using Eq. (6), first Eq. of (20), and (28), we determine the exact solution for the distribution of temperature across the shock transition region as

$$
\frac{T}{T_{1}}=\left\{1+\frac{A^{2}}{p_{1} \rho_{1}}-\frac{\rho_{1} E}{A p_{1}}\right\} \eta-\left\{\frac{A^{3}+\rho_{1}^{2} D}{A p_{1} \rho_{1}}\right\} \eta^{2}-\frac{\rho_{1} F}{A p_{1}} .
$$

From Eq. (27) together with Eq. (29), we can calculate temperature across the shock transition region with respect to the position $\xi$. Furthermore, it will be interest to determine the change-in-entropy $(\Delta S / R)_{\eta}$ as a function of $\eta$ and therefore $\xi$. In general, for the perfect gas, the change-inentropy $(\Delta \mathrm{S} / \mathrm{R})$ is given as

$$
\frac{\Delta S}{R}=\frac{\gamma}{\gamma-1} \log \left(\frac{T}{T_{1}}\right)-\log \left(\frac{p}{p_{1}}\right)
$$

using Eqns. (27), (28) and (29) into Eq. (30), we can obtain the change-in-entropy as a function of $\xi$.

\section{THICKNESS AND STRENGTH OF TRAVELLING SHOCK WAVE}

From Eq. (27), we can determine the thickness $\Delta \xi$ of the travelling shock wave between two boundary state $\eta_{1}$ and $\eta_{2}$, since $\eta_{1}$ and $\eta_{2}$ are singularity of the function $\xi=\xi(\eta)$ in the Eq. (27), therefore, we compute the values of $\xi=\xi_{1}$ at $\eta=$ $0.99 \eta_{1}+0.01 \eta_{2}$ and $\xi=\xi_{2}$ at $\eta=0.01 \eta_{1}+0.99 \eta_{2}$. The thickness of the shock wave is given in the Table 1 for the different value of $\mu$ and $M_{1}$. The thickness of the shock wave can be compared with the mean free path of the gas molecules for the validity of the continuum hypothesis. From the kinetic theory of gases one can write the viscosity as function of mean free path $[38,39]$

$$
\mu=\lambda \bar{c} \delta \rho,
$$

where, $\lambda$ is mean free path of the molecules. $\bar{c}=$ mean molecular velocity $=\sqrt{8 \rho_{1} p_{1} / \pi}$ at boundary state $\xi=-\infty$ and the theoretical values of $\delta$ vary from $1 / 3$ to 0.499 , depending on the nature of the analysis. The value used here is that of Tait. The effect of using a different value for $\delta$ (and therefore the shock-wave thickness in terms of mean free paths) in direct proportion. In this analysis we have taken the value of $\delta=$ constant $=0.35$. The mean free path of gas is computed in Table 2 for different values of viscosity under $\rho_{1}=1.225 \mathrm{~kg} / \mathrm{m}^{3}$ and $p_{1}=101325$ Pascal, for the air at $20^{\circ} \mathrm{C}$. The strength of travelling shock wave is given by

$$
Z=\frac{p_{2}-p_{1}}{p_{1}}=\gamma M_{1}^{2}\left(1-\eta_{2}\right)
$$

and is dependent on the initial Mach number $M_{1}$, uniform state $\eta_{2}$ and adiabatic index $\gamma$. The strength of shock increases with increase in initial Mach number and adiabatic index but decreases with $\eta_{2}$ at $\xi \rightarrow+\infty$.

\section{RESULTS AND DISCUSSION}

The exact solution for gas velocity has been obtained in Eq. (27) for unsteady flow of an ideal gas with viscosity under the Riemann condition by the method of travelling wave solution. The Eq. (27) gives the position $\xi$ as a one-one onto function of gas velocity $\eta$ in the range $\left(\eta_{2}, \eta_{1}\right)$, therefore, $\xi$ is monotonic function of $\eta$, so by the implicit function theorem, we can obtain gas velocity $\eta$ as a function of position $\xi$. The pressure $p / p_{1}$, temperature $T / T_{1}$ and the change-in-entropy $\Delta \mathrm{S} / \mathrm{R}$ across shock transition zone are obtained implicitely using Eq. (27) into Eqns. (28), (29), and (30), respectively, with respect to the position $\xi$. The distribution of gas velocity, the pressure, the temperature, and the change in entropy between the boundary states depends 
on initial Mach number $M_{1}$, coefficient of viscosity $\mu$, and ratio of specific heats $\gamma$ (see Figures 1, 2 and 3).

For numerical computations, we have taken $\rho_{1}=$ $1.225 \mathrm{~kg} / \mathrm{m}^{3}, p_{1}=101325$ Pascal, $\gamma=1.33,1.40$ and 1.66 , and $\mu=5 \times 10^{-6}, \mu=17.2 \times 10^{-6}$ and $\mu=$ $20 \times 10^{-6}$ Pascal second, for the air at sea level and $M_{1}=$ $1.1,1.5$ and 2 . The $\gamma=1.33,1.40$ and 1.66 corresponds to the air at more than $1000{ }^{\circ} \mathrm{C}$, air at $20{ }^{\circ} \mathrm{C}$ and ideal monoatomic gases like helium, neon and xenon at $20{ }^{\circ} \mathrm{C}$. From Eq. (23), it is found that $\eta_{1}=1$ for all value of the parameters but $\eta_{2}$ is dependent on them.

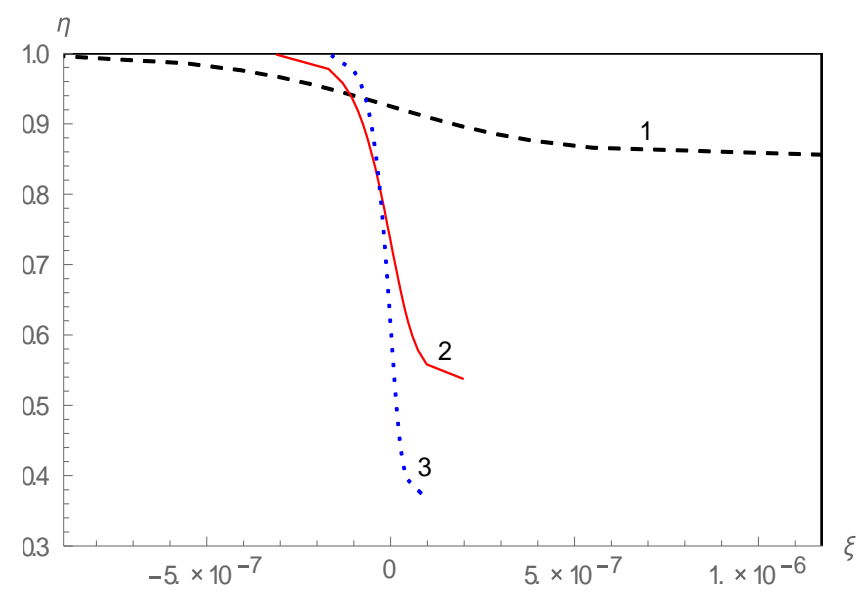

(a)

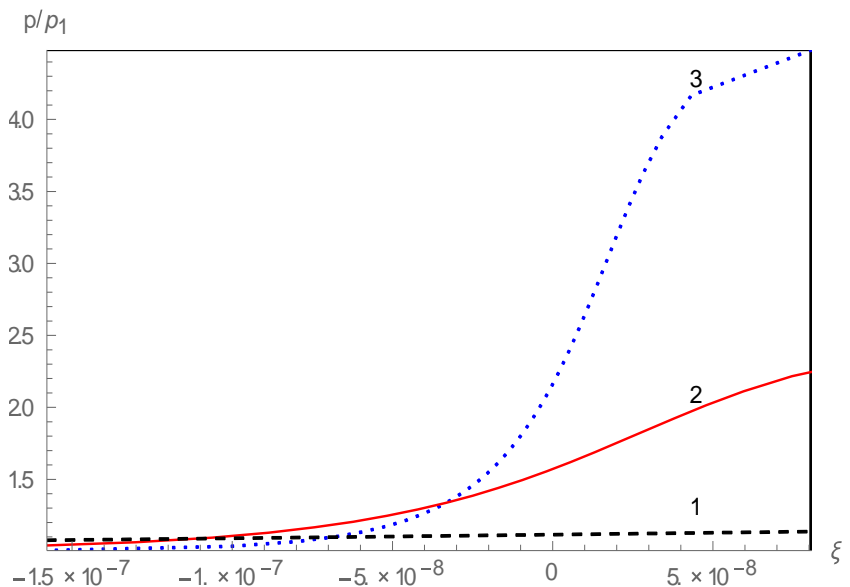

(b)

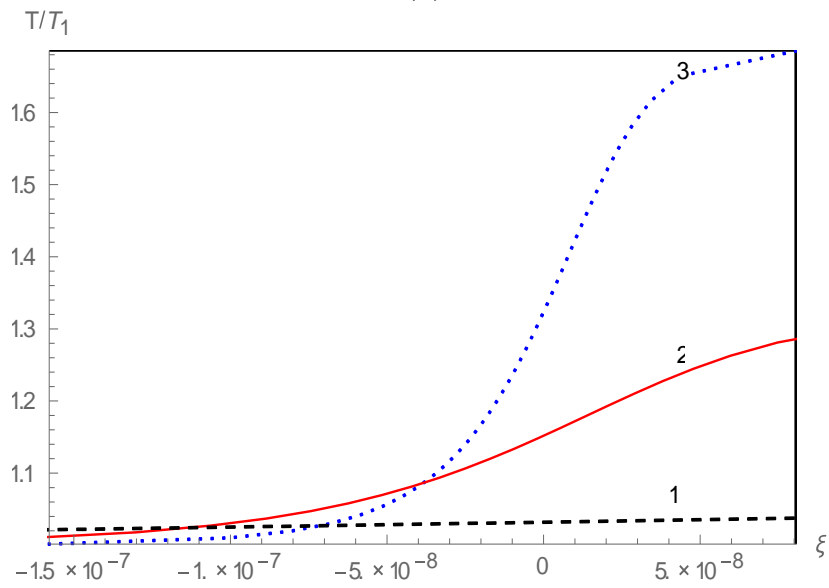

(c)

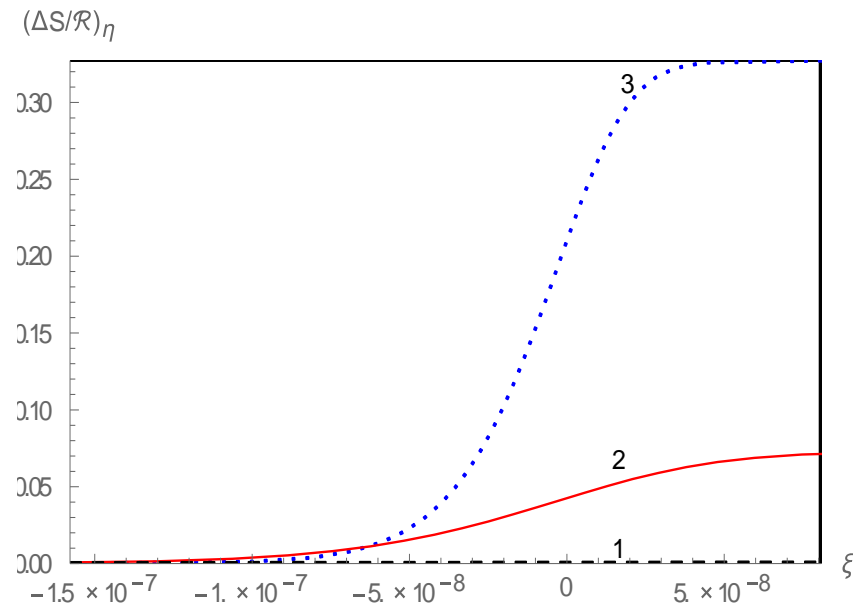

(d)

Figure 1. Variation of (a) the gas velocity $\eta$; (b) the pressure $\mathrm{p} / \mathrm{p} 1$; (c) the temperature T/T1; (d) the change-in-entropy $\Delta \mathrm{S} / \mathrm{R}$, with Mach number M1. $1 . \mathrm{M} 1=1.1,2 . \mathrm{M} 1=1.5,3$.

$$
\mathrm{M} 1=2.0
$$

The thickness $\Delta \xi$ of shock wave and shock strength have been discussed in section 5. The variation of shock wave thickness $\Delta \xi$ is given in Table 1 for various values of $\mu, M_{1}$ and $\gamma=1.40$ (air), $\rho_{1}=1.225 \mathrm{~kg} / \mathrm{m}^{3}$, and $p_{1}=101325$ Pascal. It is found that the thickness of shock wave is of the order of $10^{-6}$ meter. The mean free path $\lambda$ as a function of viscosity of the gas is obtained in the Table 2 . The inverse shock thickness $(\lambda / \Delta \xi)$ can be used as a measurement for the local Knudsen number to decide the validity of the continuum model in the study of the shock wave structure. When the local Knudsen number exceeds 0.20 [40], the continuum model must be replaced by kinetic theory. The comparison of shock wave thickness in Table 1 with mean free path in Table 2 show that continuum approach for the study of shock wave structure is valid up to a certain extent which depends on Mach number and viscosity of the gas flow (see Table 1). It is also found that the thickness become comparable to the mean free path for Mach number $M_{1} \geq$ 1.5. Therefore, taking limit of 0.20 for inverse shock thickness $(\lambda / \Delta \xi)$ in Table 2, we can conclude that this study may be valid up to Mach number $M_{1}=1.5$ due to the need of the validity of the continuum hypothesis.

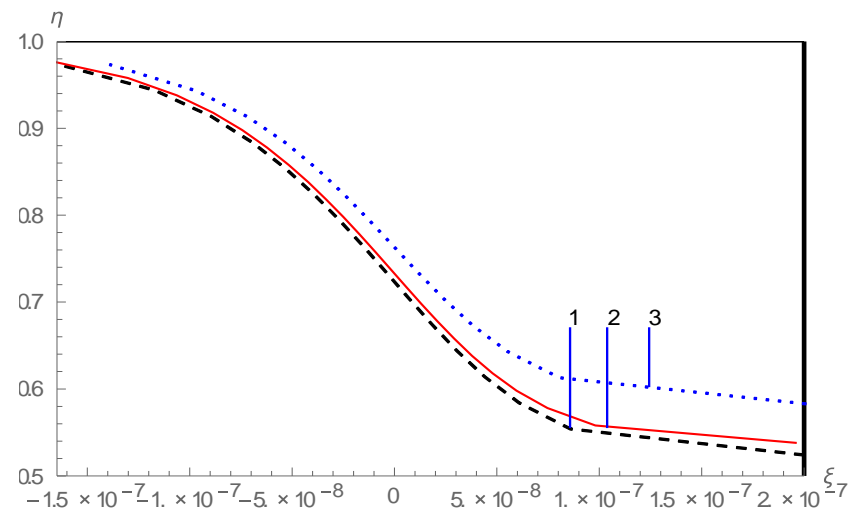

(a) 


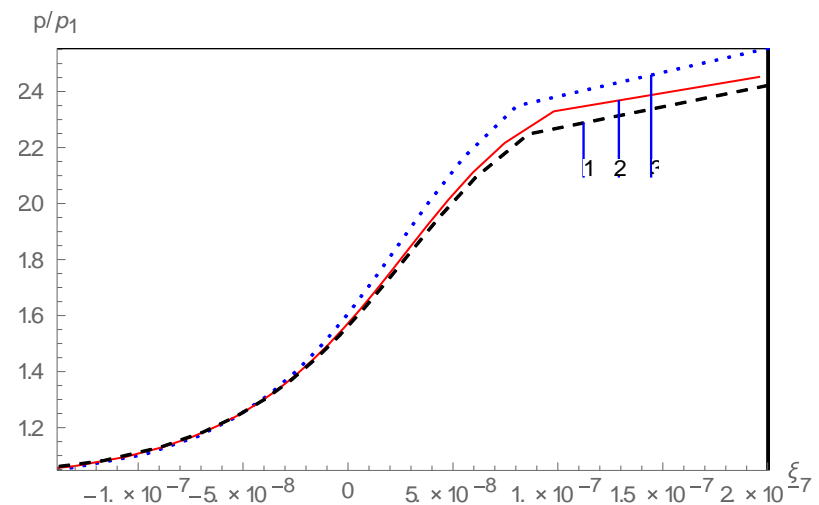

(b)

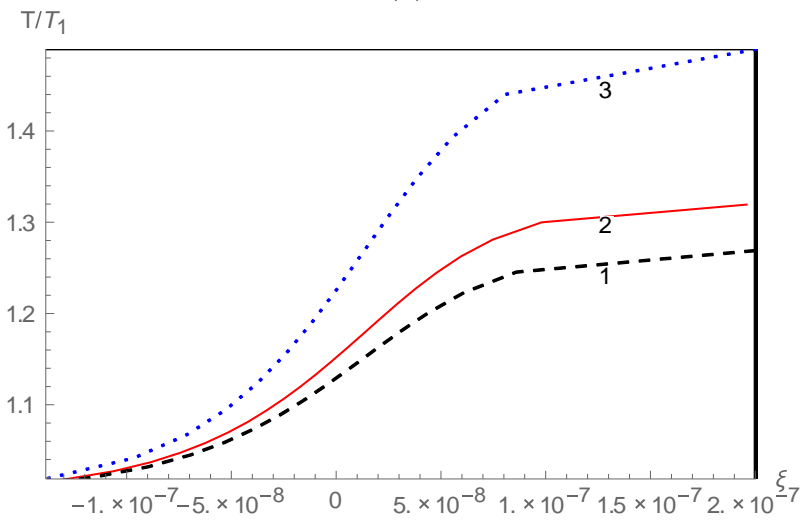

(c)

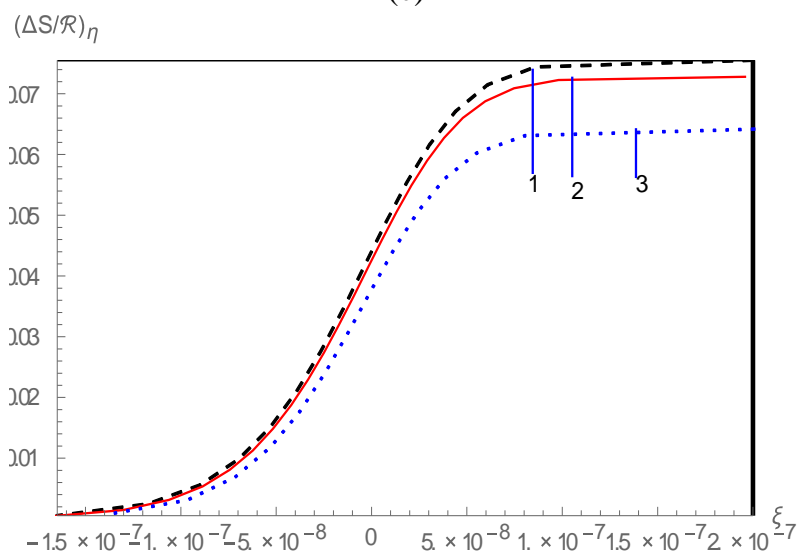

(d)

Figure 2. Variation of (a) the gas velocity $\eta$; (b) the pressure $\mathrm{p} / \mathrm{p} 1 ;$ (c) the temperature T/T1; (d) the change-in-entropy $\Delta \mathrm{S} / \mathrm{R}$, with $\gamma ; \mathbf{1} . \gamma=1.33, \mathbf{2} . \gamma=1.40, \mathbf{3} . \gamma=1.66$

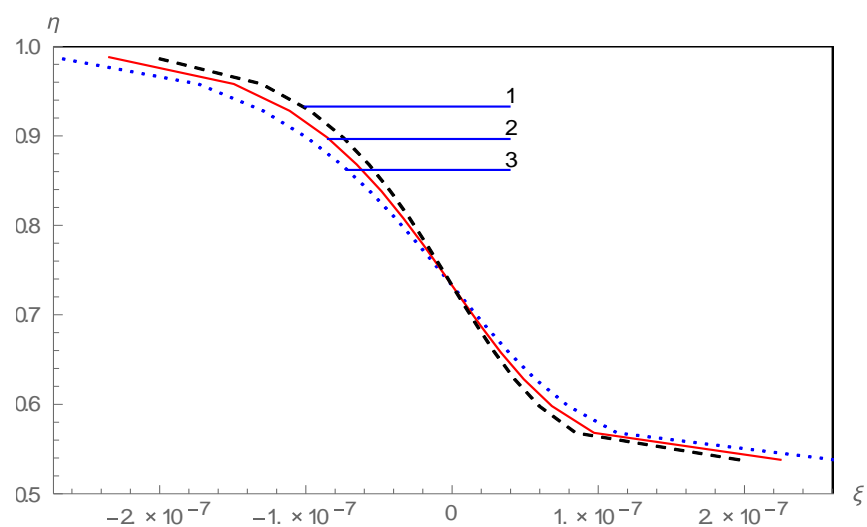

(a)

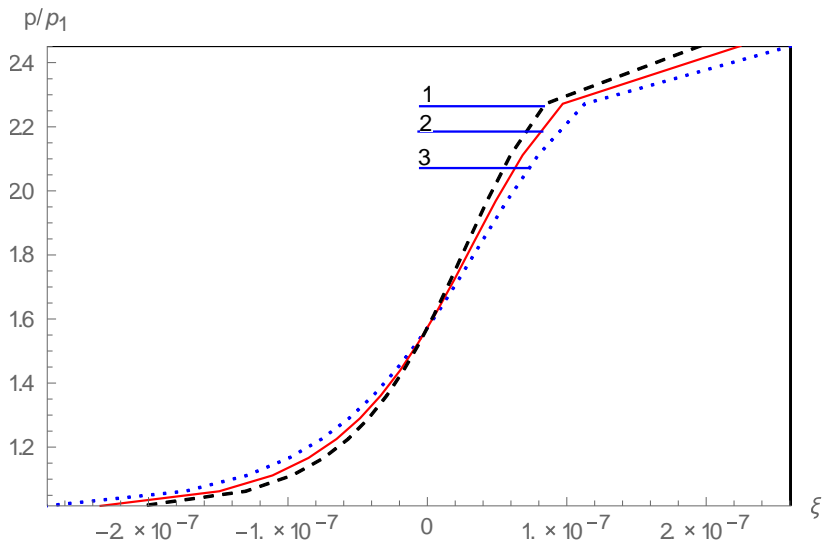

(b)

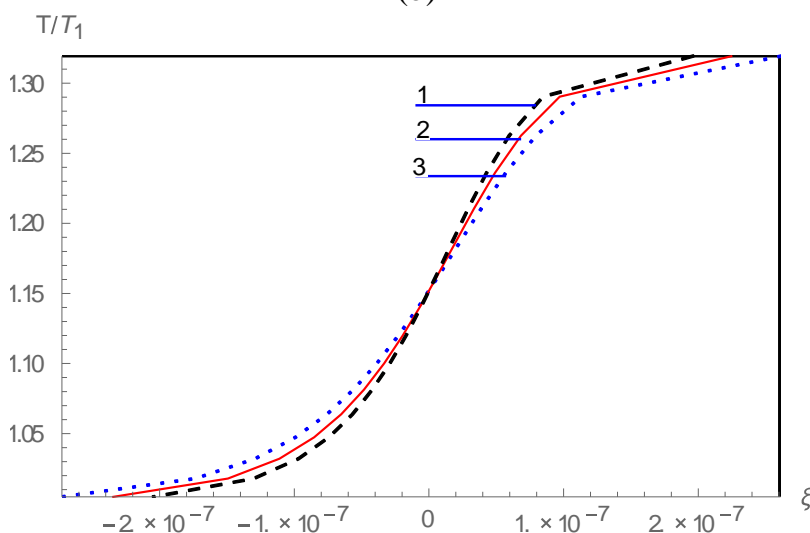

(c)

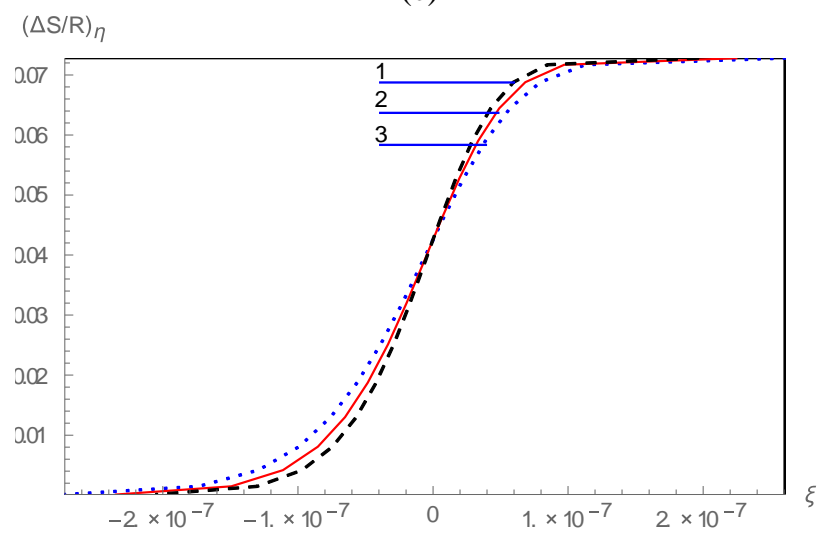

(d)

Figure 3. Variation of (a) the gas velocity $\eta$; (b) the pressure $\mathrm{p} / \mathrm{p} 1$; (c) the temperature T/T1; (d) the change-in-entropy $\Delta \mathrm{S} / \mathrm{R}$, with $\mu ; \mathbf{1} . \mu=5 \times 10^{-6}, \mathbf{2} . \mu=17.2 \times 10^{-6}, \mathbf{3} . \mu=$ $20 \times 10^{-6}$

Figures 1, 2 and 3 show that the variation of reduced flow variables $\eta, p / p_{1}, T / T_{1}$ and the change-in-entropy $\Delta S / R$ with respect to the wave variables $\xi$ for various values of parameters $\gamma, M_{1}$ and $\mu$ between the boundary $\xi=-\infty$ to $\xi=$ $+\infty$. It is evident from the Figures 1, 2 and 3 that as we move from the boundary state at $\xi=-\infty$ towards $\xi=+\infty$, the gas velocity $\eta$ decreases while pressure $p / p_{1}$, temperature $T / T_{1}$ and the change-in-entropy $\Delta S / R$ increase. The distribution of flow variables is similar to those obtained by $[30,33]$ and [41].

The effects of an increase in the value of Mach number $M_{1}$ of the gas can be summarized as: 
(i) decrease in the thickness of the shock front (see Table 1),

(ii) increase in the strength of the travelling shock wave (see Equation (32)),

(iii) decrease in the gas velocity $\eta$ (see Figure 1a). It is seen that the decrease is quite significant for large value of $M_{1}$.

(iv) Increase in the value of reduced pressure distribution $p / p_{1}$ (see Figure $1 \mathrm{~b}$ ),

(v) Increase in the value of reduced temperature $T / T_{1}$ (see Figure 1c) and change-in-entropy $\Delta \mathrm{S} / \mathrm{R}$ (see Figure 1d).

Table 1. Effect of coefficient of viscosity $\mu$ and Mach number $\mathrm{M}_{1}$ on the shock wave thickness for $\gamma=1.4, \rho_{1}=$ $1.225 \mathrm{~kg} / \mathrm{m}^{3}, p_{1}=101325$ Pascal

\begin{tabular}{|c|c|c|c|c|}
\hline $\begin{array}{c}\mu \\
{[\text { Pas s }]}\end{array}$ & $M_{1}$ & $\xi_{1}[\mathrm{~m}]$ & $\xi_{2}[\mathrm{~m}]$ & $\begin{array}{c}\Delta \xi=\xi_{1}-\xi_{2} \\
{[\mathrm{~m}]}\end{array}$ \\
\hline & 1.1 & $0.99444 \mathrm{e}-06$ & $-1.14816 \mathrm{e}-06$ & $2.14260 \mathrm{e}-06$ \\
$15 \mathrm{e}-06$ & 1.5 & $0.14685 \mathrm{e}-06$ & $-0.25978 \mathrm{e}-06$ & $0.40663 \mathrm{e}-06$ \\
& 2 & $0.05858 \mathrm{e}-06$ & $-0.14351 \mathrm{e}-06$ & $0.20209 \mathrm{e}-06$ \\
\hline & 1.1 & $1.14029 \mathrm{e}-06$ & $-1.31656 \mathrm{e}-06$ & $2.45685 \mathrm{e}-06$ \\
$17.2 \mathrm{e}-06$ & 1.5 & $0.16839 \mathrm{e}-06$ & $-0.29788 \mathrm{e}-06$ & $0.46627 \mathrm{e}-06$ \\
& 2 & $0.06718 \mathrm{e}-06$ & $-0.16456 \mathrm{e}-06$ & $0.23173 \mathrm{e}-06$ \\
\hline & 1.1 & $1.32591 \mathrm{e}-06$ & $-1.53088 \mathrm{e}-06$ & $2.85680 \mathrm{e}-06$ \\
$20 \mathrm{e}-06$ & 1.5 & $0.19580 \mathrm{e}-06$ & $-0.34638 \mathrm{e}-06$ & $0.54218 \mathrm{e}-06$ \\
& 2 & $0.07811 \mathrm{e}-06$ & $-0.19135 \mathrm{e}-06$ & $0.26946 \mathrm{e}-06$ \\
\hline
\end{tabular}

Table 2. Effect of coefficient of viscosity $\mu$ on the mean free path $\lambda$ for $\gamma=1.4, \rho_{1}=1.225 \mathrm{~kg} / \mathrm{m}^{3}, p_{1}=101325$

Pascal, $\mathrm{M}_{1}=1.5$

\begin{tabular}{|c|c|c|c|}
\hline$\mu[$ Pas s $]$ & 15 e-06 & $17.2 \mathrm{e}-06$ & $20 \mathrm{e}-06$ \\
\hline$\lambda[\mathrm{m}]$ & $0.07621 \mathrm{e}-06$ & $0.08739 \mathrm{e}-06$ & $0.11016 \mathrm{e}-06$ \\
$\lambda / \Delta \xi$ & 0.1874 & 0.1874 & 0.2032 \\
\hline
\end{tabular}

It is found that the variation of the flow variables is significant for large values of Mach number $M_{1}$. Thus the transition zone reduces into the sharp discontinuity surface for values of $M_{1} \geq 2$. It is noted that the effect of $M_{1}$ is more significant in $-5 \times 10^{-7}<\xi<\infty$.

The effects of an increase in value of adiabatic exponent $\gamma$ are:

(i) to increase the strength of the travelling shock wave (see Equation (32)),

(ii) to increase the gas velocity $\eta$ (see Figure 2a), pressure $p / p_{1}$ (see Figure $2 \mathrm{~b}$ ) and temperature $T / T_{1}$ (see Figure $2 \mathrm{c}$ ),

(iii) to decrease the change-in-entropy $\Delta S / R$ (see Figure 2d).

Like the $M_{1}$, the effect of $\gamma$ is significant in the range $-1 \times 10^{-7}<\xi<\infty$.

The effects of an increase in the coefficient of viscosity $\mu$ of the gas can be seen as:

(i) increase in the shock front thickness (see Table $1)$

(ii) increase in the mean free path of the perfect gas (see Table 2),

(iii) decrease in the velocity before the inflection point and increase after inflection point (see Figure 3a),

(iv) Increase in the pressure $p / p_{1}$, temperature $T / T_{1}$ and the change-in-entropy $\Delta \mathrm{S} / \mathrm{R}$ before the inflection point and decrease thereafter.

Therefore, the viscosity changes the variation of flow variables across the inflection point. For a gas with higher viscosity, the change in flow variables between the two boundary states is small and hence the thickness of the shock wave is large.

\section{CONCLUSIONS}

This paper investigates the structure of a moving shock wave in an unsteady flow of a perfect gas under the effect of viscosity by the method of travelling wave solution. The exact solution is obtained for the gas velocity, the pressure, the temperature and the change in entropy across a moving shock wave under the Riemann condition. The study shows that one-dimensional unsteady flow of perfect gas under the effect of viscosity and the structure of shock wave can be studied by means of travelling wave. The Riemann condition is transformed into the boundary conditions. It also shows that the motion between two boundary state depends on the viscosity, shock Mach number, ratio of specific heats of the gas and initial given data. On the basis of this work, one may draw the following conclusions:

- The thickness of the shock wave decreases with Mach number and increases with viscosity of the gas.

- The strength of shock increases with increase in initial Mach number and adiabatic index but decreases with $\eta_{2}$ at $\xi \rightarrow+\infty$

- The continuum hypothesis can be used for the study of shock wave structure for unsteady flow of the perfect gas under viscosity.

- The velocity decreases but pressure, temperature and change in entropy increase from boundary state at $-\infty$ to $+\infty$.

- The velocity decreases but the pressure, the temperature and the change in entropy incresae with Mach number in the region $0<\xi<\infty$.

- The viscosity of the gas reverses the variation of flow variables across their inflection point

- The change in flow variables across the inflection point is higher in ideally monotonic gases like helium, neon and xenon in comparison to diatomic gas like the air.

This problem may be extended by considering real gases at the place of ideal gas under non-uniform boundary states.

\section{ACKNOWLEDGEMENT}

The research of the author (Manoj Singh) is sup-ported by UGC, New Delhi, India vide letter no. Sch. No./JRF/AA/139/F-297/2012-13 dated January 22, 2013. The author (Arvind Patel) thanks to the university of Delhi, Delhi India for the R\&D grant vide letter no. RC/2015/9677 dated Oct. 15, 2015. The resreach of third author (Ruchi Bajargaan) is supported by CSIR, New Delhi vide letter no. 09/045(1264)/2012-EMR-I.

\section{REFERENCES}

[1] Fisher, R.A. (1937). The wave of advance of advantageous genes. Annuals of Human Genetics, 7(4): 355-369. 
1809.1937.tb02153.x

[2] Kolmogorov, A., Petrovsky, A., Piscounoff, N. (1988). Study of the diffusion equation with growth of the quantity of matter and its application to a biology problem. In Dynamics of Curved Fronts, Academic Press, Boston. https://doi.org/10.1016/b978-0-08092523-3.50014-9

[3] Murray, J.D. (1989). Mathematical Biology. SpringerVerlag, Berlin, New York https://doi.org/10.1016/s0092-8240(05)80386-6

[4] Mansour, M.B.A (2008). Travelling wave solutions of a nonlinear reaction-diffusion-chemotaxis model for bacterial patter formation. Appl. Math. Model., 32: 240247. https://doi.org/10.1016/j.apm.2006.11.013

[5] Apreutesei, N. (2014). Travelling wave solutions of reaction-diffusion equations arising in atherosclerosis models. Math., 2: 83-95. https://doi.org/10.3390/math2020083

[6] Marchant, B.P., Norbury, J., Perumpanani, A.J. (2000). Travelling shock wave arising in a model of malignant invasion. Siam. J. Appl. Math., 60(2): 265-476. https://doi.org/10.1137/s0036139998328034

[7] Marchant, B.P., Norbury, J., Sherratt, J.A. (2001). Travelling wave solutions to a haptotaxis-dominated model of malignant invasion. Inst. of Phys. Publ. Nonlinearty, 14: 1653-1671. https://doi.org/10.1088/0951-7715/14/6/313

[8] Camacho, V., Guy Robert, D., Jacobsen, J. (2008). Travelling waves and shock in a viscoelastic generalization of burger' equation. Siam J. Appl. Math., 68(5): 1316-1332. https://doi.org/10.1137/070687840

[9] Erbay, H.A., Sengul, Y. (2015). Traveling waves in one-dimensional non-linear models of strain-limiting viscoelasticity. Int. J. Non-Linear Mech., 77: 61-68. https://doi.org/10.1016/j.ijnonlinmec.2015.07.005

[10] Roshid, M., Bashar, H. (2019). Breather wave and kinky periodic wave solutions of one-dimensional Oskolkov equation. Mathematical Modelling of Engineering Problems, 6(3): 460-466. https://doi.org/10.18280/mmep.060319

[11] Glimm, J. (1963). Solutions in the large for nonlinear hyperbolic systems of equations. Comm. Pure. Appl. Math., 18

$697-715$ https://doi.org/10.1002/cpa.3160180408

[12] Courant, R., Friedrichs, K.O. (1999). Supersonic flow and shock waves. Interscience Publishers, New York. https://doi.org/10.1007/978-1-4684-9364-1

[13] Lax, P.D. (1957). Hyperbolic systems of conservation laws II. Comm. Pure. Appl. Math., 10: 537-566. https://doi.org/10.1002/cpa.3160100406

[14] Godunov, S.K. (1976). Numerical solution of multidimensional problems in gasdynamics. Nauka Press, Moscow. https://doi.org/10.1086/ahr/85.2.431

[15] Smoller, J. (1994). Shock waves and reaction diffusion equations. Springer Verlag (2nd ed.), New York. https://doi.org/10.1007/978-1-4612-0873-0_14

[16] Roe, P.L. (1981). Approximate Riemann solvers, parameter vectors, and difference schemes. J. Comput. Phy., 43: 357-372. https://doi.org/10.1016/00219991(81)90128-5

[17] Colella, P., Glaz, H.M. (1985). Efficient solution algorithms for the Riemann problem for real gases. J. Comput. $\quad$ Phy., $\quad 59$ : 264-289. https://doi.org/10.1016/0021-9991(85)90146-9
[18] Ambika, K., Radha, R. (2016). Riemann problem in non-ideal gas dynamic. Indian J. Pure Appl. Math., 47(3): 501-521. https://doi.org/10.1007/s13226-0160200-9

[19] Rankine, W.J.M. (1870). On the thermodynamic theory of waves of finite longitudinal disturbances. Philos. Trans. R. Soc. Lond., 160: 277-288. https://doi.org/10.1098/rstl.1870.0015

[20] Rayleigh, L. (1910). Arial plane waves of finite amplitude. Proc. R. Soc. Lond. A, 84: 247-284. https://doi.org/10.1098/rspa.1910.0075

[21] Taylor, G.I. (1910). The conditions necessary for discontinuous motion in gases. Proc. R. Soc. Lond. A, 84: 371-377. https://doi.org/ 10.1098/rspa.1910.0081

[22] Lamb, H. (1945). Hydrodynamics. 6th Rev. Ed., Dover Publications, New York. https://doi.org/10.1017/s0368393100132535

[23] Becker, R. (1922). Impact waves and detonation. Z. Phys., transtation N.A.C.A.- $\quad$ T.M., 8. https://doi.org/10.1007/bf01329605

[24] Thomas, L.H. (1944). Note on Becker's theory of the shock front. J. Chem. Phys., 12: 449-453. https://doi.org/10.1063/1.1723889

[25] Meyerhoff, L., Reissner, H.J. (1949). The standing, onedimensional shock wave under the influence of temperature-dependent viscosity. Heat Conduction and Specific Heat. PIBAL Report, Vol. 150.

[26] Puckett, A.E., Stewart, H.J. (1950). The thickness of a shock wave in air. Quart. Appl. Math., 7: 457-463. https://doi.org/10.1090/qam/33711

[27] Thompson, P.A., Lambrakis, K.C. (1973). Negative shock waves. J. Fluid Mech., 60: 187-208. https://doi.org/10.1017/S002211207300011X

[28] Cramer, M.S., Crickenberger, A.B. (1991). The dissipative structure of shock waves in dense gases. J. Fluid Mech., 223: 325-355. https://doi.org/10.1017/S0022112091001441

[29] Johnson, B.M. (2013). Analytical shock solutions at large and small Prandtl number. J. Fluid Mech., 726: 112. https://doi.org/10.1017/jfm.2013.262

[30] Yadav, H.C., Anand, R.K. (2011). Propagation of shock waves in a viscous medium. Phys. Scr., 83: 065-402. https://doi.org/10.1088/00318949/83/06/065402

[31] Anand, A.K., Yadav, H.C. (2014). On the structure of MHD shock waves in a viscous non-ideal gas. Theor. Comput. Fluid Dyn., 28: 369-376. https://doi.org/10.1007/s00162-014-0320-y

[32] Myong, R.S. (2014). Technical note for analytical solutions of shock structure thickness and asymmetry in Navier-Stokes/Fourier Framework. AIAA J., 52(5): 1075-1080. https://doi.org/10.2514/1.J052583

[33] Patel, A., Manoj, S. (2019). Exact solution of shock wave structure in a non-ideal gas under constant and variable coefficient of viscosity and heat conductivity. Shock Waves, 29: 427-439. https://doi.org/10.1007/s00193-018-0855-8

[34] Singh, M., Patel, A. (2019). Shock wave structure in a non-ideal gas under temperature and density-dependent viscosity and heat conduction. Theor. Comput. Fluid Dyn., 1-23. https://doi.org/10.1007/s00162-019-00505y

[35] Singh, M., Patel, A., Bajargaan, R. (2016). Study of a one-dimensional unsteady gas dynamic problem by Adomain's decomposition method. Int. J. Appl. Math, 
29(6): 775-794. http://dx.doi.org/10.12732/ijam.v29i6.8

[36] Bajargaan, R., Patel, A., Singh, M. (2017). Homotopy analysis method for one dimensional unsteady adiabatic gas flow. Int. J. Pure Appl. Math, 115(4): 673-692. https://doi.org/10.12732/ijpam.v115i4.3

[37] Whitham, G.B. (1974). Linear and nonlinear waves. A Wiley-Interscience Publication, John Wiley and sons INC., New York. https://doi.org/10.1017/s0022112078211676

[38] Loeb, L.B. (1927). Kinetic Theory of Gases. McGraw-
Hill Book Company, Inc.

[39] Jeans, J. (1967). An Introduction to the Kinetic Theory of Gases. Cambridge at the university press, New York.

[40] Bird, G.A. (1994). Molecular Gas Dynamics and the Direct Simulation of Gas Flows. 2nd edition. Clarendon Press, Oxford.

[41] Morduchow, M. Libby, P.A. (1949). On a complete solution of the one-dimensional flow equations of viscous, heat conducting, compressible gas. J. Aeronaut. Sci., 16: 674-684. https://doi.org/10.2514/8.11882 